\title{
VIABILITY AND INVARIANCE FOR DIFFERENTIAL GAMES WITH APPLICATIONS TO HAMILTON-JACOBI-ISAACS EQUATIONS
}

\author{
PIERRE CARDALIAGUET \\ CEREMADE, Université Paris-Dauphine \\ 75775 Paris Cedex 16, France \\ E-mail: cardiala@paris9.dauphine.fr \\ SŁAWOMIR PLASKACZ \\ Department of Mathematics and Informatics, Nicholas Copernicus University \\ Chopina 12/18, 87-100 Toruń, Poland \\ E-mail: plaskacz@mat.uni.torun.pl
}

1. Introduction We consider a differential game with dynamics given by $x^{\prime}(t)=$ $f(t, x(t), y, z)$. By $x\left(\cdot ; t_{0}, x_{0}, y(\cdot), z(\cdot)\right)$ we denote the solution of the Cauchy problem

$$
\left\{\begin{array}{l}
x^{\prime}(t)=f(t, x(t), y(t), z(t)) \quad \text { for a.e. } t \in[0, T] \\
x\left(t_{0}\right)=x_{0}
\end{array}\right.
$$

where $y:[0, T] \rightarrow Y, z:[0, T] \rightarrow Z$ are measurable controls (open loops) of player I and II, respectively and $Y, Z$ are compact metric spaces. Let $M_{t}=\{y:[t, T] \rightarrow$ $Y \mid y$ is measurable $\}$ and $N_{t}=\{z:[t, T] \rightarrow Z \mid z$ is measurable $\}$. We say that a map $\alpha: N_{t} \rightarrow M_{t}$ is a strategy if for every controls $z_{1}, z_{2} \in N_{t}$ such that

$$
z_{1}(s)=z_{2}(s) \quad \text { for a.a. } s \in[t, \tau]
$$

we have

$$
\alpha\left(z_{1}\right)(s)=\alpha\left(z_{2}\right)(s) \quad \text { for a.a. } s \in[t, \tau]
$$

Let $\Gamma_{t}$ denotes the set of all strategies $\alpha: N_{t} \rightarrow M_{t}$.

We define the value function $U:[0, T] \times \mathbf{R}^{n} \rightarrow \mathbf{R}$ by:

$$
U\left(t_{0}, x_{0}\right)=\sup _{\alpha \in \Gamma_{t_{0}}} \inf _{z \in N_{t_{0}}} g\left(x\left(T ; t_{0}, x_{0}, \alpha(z), z\right)\right),
$$

where $g: \mathbf{R}^{n} \rightarrow \mathbf{R}$ is a terminal time cost function. Hamiltonian $H:[0, T] \times \mathbf{R}^{n} \times \mathbf{R}^{n} \rightarrow \mathbf{R}$

1991 Mathematics Subject Classification: Primary 90D25; Secondary 49L25, 35F20.

The paper is in final form and no version of it will be published elsewhere. 
is determined by the right hand side $f(t, x, y, z)$

$$
H(t, x, p)=\min _{z \in Z} \max _{y \in Y}\langle f(t, x, y, z), p\rangle .
$$

If the function $U$ is differentiable in its domain then it satisfies Isaacs (Hamilton-JacobiIsaacs) equation:

$$
U_{t}+H\left(t, x, U_{x}\right)=0 .
$$

But it can happen that $U$ is not differentiable. Crandall and Lions in [6] introduced viscosity solutions for first order PDE's, and proved existence and uniqueness of such solutions for wide class of Hamilton-Jacobi equations. Evans and Souganidis in [8] proved that $U$ is a viscosity solution of Isaacs equation. Our aim is to prove that if a Lipschitz continuous function $W:[0, T] \times \mathbf{R}^{n} \rightarrow \mathbf{R}$ is a viscosity solution of Isaacs equation and satisfies the condition $W(T, \cdot)=g(\cdot)$ then $W=U$. The result is not new. We provide a new proof using only elementary tools. Namely, we shall use results concerning invariant solutions of differential games with respect to a constraint subset of the extended phase space. The technic was invented by H. Frankowska in [9] for Hamilton-Jacobi-Bellman equations genereted by control systems, or equivalently, by differential inclusions. In [9], the viability and invariance results for differential inclusion was used to obtain uniqueness of solution.

In the second section we adopt results concerning invariant solutions of differential inclusions to the case of differential games. In the third section we compare some equivalent concept of viscosity solutions and we prove the uniqueness of viscosity solution to Hamilton-Jacobi-Isaacs equation.

2. Viability and invariance for differential games. We consider a set-valued map $P:[0, T] \rightsquigarrow \mathbf{R}^{n}$, i.e. $P(t) \subset \mathbf{R}^{n}$ and $P(t) \neq \emptyset$ for every $t \in[0, T]$, regarded as a time dependent constraint set or a tube of constraint. A tube $P$ is called absolutely continuous if there exists an integrable function $\mu:[0, T] \rightarrow[0,+\infty)$ such that for every $t_{1}<t_{2}$ we have

$$
d_{H}\left(P\left(t_{1}\right), P\left(t_{2}\right)\right) \leq \int_{t_{1}}^{t_{2}} \mu(s) d s
$$

where $d_{H}(\cdot, \cdot)$ is the Hausdorf distance,

$$
\begin{aligned}
d_{H}(A, B) & =\inf \left\{\varepsilon>0 \mid A \subset O^{\varepsilon}(B) \text { and } B \subset O^{\varepsilon}(A)\right\}, \\
O^{\varepsilon}(A) & =\{x \mid \exists a \in A,\|x-a\|<\varepsilon\} .
\end{aligned}
$$

Using Hausdorff distance we extend the notion of nonexpansive, Lipschitz continuous or continuous maps into the case of set-valued maps. Given a closed subset $K$ of Euclidean space $E$ the Bouligand contingent cone $T_{K}(x)$ to $K$ at $x \in K$ is defined by

$$
T_{K}(x)=\left\{e \in E \mid \liminf _{h \rightarrow 0^{+}} \frac{\operatorname{dist}(x+h e, K)}{h}=0\right\} .
$$

For $T \subset E$ we let $T^{\perp}$ for the polar cone to $T$,

$$
T^{\perp}=\{w \in E \mid\langle w, v\rangle \leq 0 \text {, for every } v \in T\} .
$$

We set

$$
N_{K}^{0}(x)=T_{K}^{\perp}(x)
$$

and say that $N_{K}^{0}(x)$ is the normal cone to $K$ at $x \in K$. 
We assume $f:[0, T] \times \mathbf{R}^{n} \times Y \times Z \rightarrow \mathbf{R}^{n}$ is uniformly continuous with

$$
\begin{aligned}
\|f(t, x, y, z)\| & \leq C \\
\left\|f(t, x, y, z)-f\left(t_{1}, x_{1}, y, z\right)\right\| & \leq L\left(\left|t_{1}-t\right|+\left\|x_{1}-x\right\|\right) ;
\end{aligned}
$$

for some constants $C, L$ and all $t, t_{1} \in[0, T], x, x_{1} \in R^{n}, y \in Y, z \in Z$.

The approach to Hamilton-Jacobi-Bellman equations in [9] bases on viability and invariance results for differential inclusions. The role of viability result will be played by the following theorem.

THEOREM 1. We assume that $P:[0, T] \rightsquigarrow \mathbf{R}^{n}$ is absolutely continuous and $f$ : $[0, T] \times \mathbf{R}^{n} \times Y \times Z \rightarrow \mathbf{R}^{n}$ additionally satisfies

$$
\forall(t, x, z) \in[0, T] \times R^{n} \times Z\{f(t, x, y, z) \mid y \in Y\} \text { is convex } .
$$

and for all $t \in(0, T), x \in P(t)$ we have

(4) $\forall\left(n_{t}, n_{x}\right) \in N_{\operatorname{Graph}(P)}^{0}(t, x), \forall z \in Z, \exists y \in Y,\left\langle\left(n_{t}, n_{x}\right),(1, f(t, x, y, z))\right\rangle \leq 0$.

Then for every $t_{0} \in(0, T)$ and $x_{0} \in P\left(t_{0}\right)$

$$
\exists \alpha \in \Gamma_{t_{0}}, \forall z(\cdot) \in N_{t_{0}}, \forall t \in\left[t_{0}, T\right], x\left(t ; t_{0}, x_{0}, \alpha(z), z\right) \in P(t) .
$$

The proof of Theorem 1 makes use of a viability result for differential inclusions and a nonexpansive selection theorem in ultrametric spaces. First, we recall a viability result for differential inclusion in an appropriate version.

TheOREM 2 (Theorem 3.1 in [10]). Assume that a nonempty closed valued tube $P$ : $[0, T] \rightsquigarrow R^{n}$ is absolutely continuous and a set-valued map $F:[0, T] \times R^{n} \rightsquigarrow R^{n}$ satisfies the following conditions:

(5) $F(t, x)$ is nonempty closed convex,

(6) $\quad x \rightsquigarrow F(t, x)$ is upper semicontinuous for almost all $t \in[0, T]$,

(7) $F(\cdot, \cdot)$ is $\mathcal{L} \times \mathcal{B}$ measurable,

(8) $\exists \mu \in L^{1}(0, T)\|F(t, x)\| \leq \mu(t)(1+\|x\|)$ for a.a. $t \in[0, T]$ and all $x \in R^{n}$.

Then the following statements are equivalent:

(i) There exists $C \subset[0, T]$ of full measure such that for all $t \in C, x \in P(t)$

$$
(\{1\} \times F(t, x)) \cap \overline{\mathrm{co}}\left(T_{\mathrm{Graph}(P)}(t, x)\right) \neq \emptyset .
$$

(ii) For every $t_{0} \in[0, T)$ and every $x_{0} \in P\left(t_{0}\right)$ there exists an absolutely continuous solution $x:\left[t_{0}, T\right] \rightarrow R^{n}$ of

$$
\left\{\begin{array}{l}
x^{\prime} \in F(t, x), \\
x\left(t_{0}\right)=x_{0}, \\
x(t) \in P(t) \quad \text { for all } t \in\left[t_{0}, T\right] .
\end{array}\right.
$$

A metric $\rho$ in a space $M$ is an ultrametric if it satisfies strong triangle inequality

$$
\rho(x, z) \leq \max (\rho(x, y), \rho(y, z)) .
$$

We say that a subset $K$ of an ultrametric space $M$ is $(*)$-closed if for every sequence $\left\{y_{n}\right\} \subset K$ and every sequence $\left\{c_{n}\right\}\left(c_{n} \geq c_{n+1} \geq 0\right)$ such that $\rho\left(y_{n}, y_{n+1}\right) \leq c_{n}$, there is $\bar{y} \in K$ such that $\rho\left(\bar{y}, y_{n}\right) \leq c_{n}$, for every $n$. 
Lemma 3 (Nonexpansive selection). If $A: N \rightsquigarrow M$ is a nonexpansive set-valued map from an ultrametric space $\left(N, \rho_{N}\right)$ into an ultrametric space $\left(M, \rho_{M}\right)$ with nonempty $(*)$ closed values, then there exists a nonexpansive selection $\alpha: N \mapsto M$ of $A$.

Remark 4 . Given $y_{1}, y_{2} \in M_{t_{0}}$ we define

$$
\rho\left(y_{1}, y_{2}\right)=T-\sup \left\{t \in\left[t_{0}, T\right] \mid y_{1}(s)=y_{2}(s) \text { for a.a. } s \in\left[t_{0}, t\right]\right\} .
$$

It is easy to see that $\left(M_{t_{0}}, \rho\right)$ is an ultrametric space. Moreover, a strategy $\alpha: N_{t_{0}} \rightarrow M_{t_{0}}$ is nothing else as a nonexpansive map in the meaning of the ultrametric $\rho$.

The proof of Lemma 3 will be given in a forthcoming paper of the authors.

Proof of Theorem 1. Fix $t_{0} \in[0, T], x_{0} \in P\left(t_{0}\right)$ and $\widetilde{z}(\cdot) \in N_{t_{0}}$. We define a set-valued map $F_{\widetilde{z}(\cdot)}(t, x)=\{f(t, x, y, \widetilde{z}(t) \mid y \in Y\}$. By the regularity of $f$, the set-valued map $F_{\widetilde{z}(\cdot)}$ satisfies $(5),(6),(7),(8)$. By the separation theorem and (4), we have for every $t \in(0, T)$ and $x \in P(t)$

$$
\forall z \in Z \exists y \in Y(1, f(t, x, y, z)) \in \overline{\mathrm{co}}\left(T_{\mathrm{Graph}(P)}(t, x)\right) .
$$

Thus $F_{\widetilde{z}(\cdot)}$ satisfies statement (i) in Theorem 2. Therefore there exists an absolutely continuous solution $\widetilde{x}:\left[t_{0}, T\right] \rightarrow R^{n}$ of the differential inclusion $\widetilde{x}^{\prime}(t) \in F_{\widetilde{z}(\cdot)}(t, \widetilde{x}(t))$ such that $\widetilde{x}\left(t_{0}\right)=x_{0}$ and $\widetilde{x}(t) \in P(t)$, for every $t \in\left[t_{0}, T\right]$. By Theorem 8.2.10 in [3], there exists a measurable map $\widetilde{y}:\left[t_{0}, T\right] \rightarrow Y$ such that $x\left(t ; t_{0}, x_{0}, \widetilde{y}(\cdot), \widetilde{z}(\cdot)\right)=\widetilde{x}(t)$ for $t \in\left[t_{0}, T\right]$.

We define a set-valued map $A: N_{t_{0}} \rightsquigarrow M_{t_{0}}$ by:

$$
A(z(\cdot))=\left\{y(\cdot) \in M_{t_{0}} \mid x\left(t ; t_{0}, x_{0}, y(\cdot), z(\cdot)\right) \in P(t) \text { for } t \in\left[t_{0}, T\right]\right\} .
$$

We have shown that the values of the map $A$ are nonempty. Now we verify that the map $A$ satisfies the remaining assumptions of Lemma 3 .

Let $z_{1}, z_{2} \in N_{t_{0}}$ and $y_{1} \in A\left(z_{1}\right)$. We set $t_{1}=T-\rho\left(z_{1}, z_{2}\right)$ and $x_{1}=x\left(t_{1} ; t_{0}, x_{0}, y_{1}, z_{1}\right)$. We have $x_{1} \in P\left(t_{1}\right)$. By (10) and Theorem 2, there exists a solution $\widehat{x}:\left[t_{1}, T\right] \rightarrow R^{n}$ of a differential inclusion $\widehat{x}^{\prime}(t) \in F_{z_{2}}(t, \widehat{x}(t))$ such that $\widehat{x}\left(t_{1}\right)=x_{1}$ and $\widehat{x}(t) \in P(t)$ for $t \in\left[t_{1}, T\right]$, where $F_{z_{2}}(t, x)=\left\{f\left(t, x, y, z_{2}(t)\right): y \in Y\right\}$. By Theorem 8.2.10 in [3], there exists a measurable map $y_{3}:\left[t_{1}, T\right] \rightarrow Y$ such that $x\left(t ; t_{1}, x_{1}, y_{3}, z_{2}\right)=\widehat{x}(t)$ for $t \in\left[t_{1}, T\right]$. Setting

$$
y_{2}(t)= \begin{cases}y_{1}(t) & \text { for } t \in\left[t_{0}, t_{1}[\right. \\ y_{3}(t) & \text { for } t \in\left[t_{1}, T\right]\end{cases}
$$

we get $y_{2} \in A\left(z_{2}\right)$ such that $\rho\left(y_{1}, y_{2}\right) \leq \rho\left(z_{1}, z_{2}\right)$, which means that the map $A$ is nonexpansive.

Now, we show that the set $A(z)$ is $(*)$-closed, for every $z \in N_{t_{0}}$. Let $0 \leq \ldots \leq c_{k+1} \leq$ $c_{k} \leq \ldots \leq c_{1} \leq T-t_{0}, c=\lim _{k \rightarrow \infty} c_{k}$ and $y_{k} \in A(z)$ satisfy $\rho\left(y_{k}, y_{k+1}\right) \leq c_{k}$. We set $t_{k}=T-c_{k}$. Obviously, we have $x\left(t ; t_{0}, x_{0}, y_{k}, z\right)=x\left(t ; t_{0}, x_{0}, y_{k+1}, z\right)$ for $t \in\left[t_{0}, t_{k}\right]$. We define a map $y_{\infty}:\left[t_{0}, T-c[\rightarrow Y\right.$ by

$$
y_{\infty}(t)= \begin{cases}y_{1}(t) & \text { for } t \in\left[t_{0}, t_{1}[\right. \\ y_{k}(t) & \text { for } t \in\left[t_{k-1}, t_{k}[\text { and } k=2,3, \ldots\right.\end{cases}
$$

We set $x_{\infty}=\lim _{t \rightarrow(T-c)}-x\left(t ; t_{0}, x_{0}, y_{\infty}, z\right)$. It is easy to check that $x_{\infty} \in P(T-c)$. By (10) and Theorem 2, there exists a solution $\bar{x}:[T-c, T] \rightarrow R^{n}$ of a differential 
inclusion $\bar{x}^{\prime}(t) \in F_{z}(t, \bar{x}(t))$ such that $\bar{x}(T-c)=x_{\infty}$ and $\bar{x}(t) \in P(t)$ for $t \in[T-c, T]$. By Theorem 8.2.10 in [3], there exists a measurable map $\bar{y}:[T-c, T] \rightarrow Y$ such that $x\left(t ; T-c, x_{\infty}, \bar{y}, z\right)=\bar{x}(t)$ for $t \in[T-c, T]$. Setting

$$
y(t)= \begin{cases}y_{\infty}(t) & \text { for } t \in\left[t_{0}, T-c[\right. \\ \bar{y}(t) & \text { for } t \in[T-c, T]\end{cases}
$$

we get $y \in A(z)$ such that $\rho\left(y_{k}, y\right) \leq c_{k}$, which means that the set $A(z)$ is $(*)$-closed.

Now, by Lemma 3, there exists a nonexpansive selection $\alpha: N_{t_{0}} \rightarrow M_{t_{0}}$ of $A$, which is the desired strategy.

Now, we shall study the problem of invariance for differential games.

Lemma 5. Let $K$ be a closed subset of $R^{n}$ and $\sigma>0, M>0$ given constants. Then for every $\varepsilon>0$, there exists $\delta>0$ such that for every pairs of points $(k, q) \in K \times R^{n}$ such that $|q-k|=\operatorname{dist}(q, K)>\sigma$, and for any measurable function $g:[0,1] \longrightarrow R^{n}$ such that $|g(t)| \leq M \mid$ and $\langle g(t), q-k\rangle \leq 0$ for almost all $t \in[0,1]$, we have

$$
\forall h \in(0, \delta], \operatorname{dist}\left(q+\int_{0}^{h} g(t) d t, K\right)<|q-k|+h \cdot \varepsilon
$$

Pro of. We have $\left|q+\int_{0}^{h} g(t) d t-k\right|^{2}=|q-k|^{2}+\left|\int_{0}^{h} g(t) d t\right|^{2}+2\left\langle q-k, \int_{0}^{h} g(t) d t\right\rangle \leq$ $|q-k|^{2}+h^{2} M^{2}$. Thus

$$
\left|q+\int_{0}^{h} g(t) d t-k\right|-|q-k|=\frac{\left|q+\int_{0}^{h} g(t) d t-k\right|^{2}-|q-k|^{2}}{\left|q+\int_{0}^{h} g(t) d t-k\right|+|q-k|} \leq \frac{h^{2} M^{2}}{\sigma}
$$

which completes the proof.

Theorem 6. Let $K$ be a closed subset of $\mathbf{R}^{n}$ and $x_{0} \in K$. Suppose that $f: \mathbf{R}^{n} \times$ $Y \times Z \longrightarrow \mathbf{R}^{n}$ is a continuous bounded map and $f(\cdot, y, z)$ is Lipschitz continuous with a constant $L$ which does not depend to $y, z$. If

$$
\forall(x, p) \in K \times \mathbf{R}^{n}|p-x|=\operatorname{dist}(p, K) \exists z \in Z \forall y \in Y\langle f(x, y, z), p-x\rangle \leq 0
$$

then

$$
\forall \varepsilon>0 \forall \alpha \in \Gamma_{0} \exists z \in N_{0} \operatorname{dist}\left(x\left(T ; 0, x_{0}, \alpha(z), z\right), K\right)<\varepsilon .
$$

Proof. Fix $\alpha \in \Gamma_{0}$. The proof is provided in two steps.

STEP 1. Denote by $R$ the upper bound of $f$ and fix $\gamma>0$. Let $\Pi=\left(0=t_{0}<t_{1}<\right.$ $\left.\ldots<t_{k}=T\right)$ be a division of $[0, T]$ such that the diameter $\operatorname{diam}(\Pi)$ of $\Pi$ satisfies the following estimations:

$$
\begin{gathered}
\operatorname{diam}(\Pi) \cdot R<\gamma / 2, \\
\operatorname{diam}(\Pi) \leq \delta,
\end{gathered}
$$

where $\delta$ is chosen by Lemma 5 for $\varepsilon=\gamma$ and $\sigma=\gamma / 2$. We construct two maps $z:[0, T] \rightarrow Z$ and $p:[0, T] \rightarrow R^{n}$ by extending its onto successive intervals of the division $\Pi$. Suppose that $z(\cdot), p(\cdot)$ are already defined on $\left[0, t_{i}\right]$ and $p(0)=x_{0}$. There exists $p_{i} \in K$ such that $\left|p_{i}-p\left(t_{i}\right)\right|=\operatorname{dist}\left(p\left(t_{i}\right), K\right)$. By assumption (11), there is $z_{i} \in Y$ such that $\left\langle f\left(p_{i}, y, z_{i}\right), p\left(t_{i}\right)-p_{i}\right\rangle \leq 0$, for every $y \in Y$. We define

$$
w_{i}(t)= \begin{cases}z(t) & \text { for } t \leq t_{i} \\ z_{i} & \text { for } t>t_{i}\end{cases}
$$


Now, we extend $z(\cdot), p(\cdot)$ setting $z(t)=z_{i}$ and $p(t)=p\left(t_{i}\right)+\int_{t_{i}}^{t} f\left(p_{i}, \alpha\left(w_{i}\right)(s), w_{i}(s)\right) d s$ for $t \in\left(t_{i}, t_{i+1}\right]$.

We have $\left|p\left(t_{i}\right)-p_{i}\right| \leq \gamma\left(t_{i}+1\right)$.

Indeed, if $\left|p\left(t_{i}\right)-p_{i}\right|<\gamma / 2$, then using (12) we get $\left|p\left(t_{i+1}\right)-p_{i+1}\right|<\gamma$. If $\left|p\left(t_{i}\right)-p_{i}\right| \geq$ $\gamma / 2$, then by (13), we obtain the estimation dist $\left(p\left(t_{i+1}\right), K\right) \leq\left|p\left(t_{i}\right)-p_{i}\right|+\gamma\left(t_{i+1}-t_{i}\right) \leq$ $\gamma\left(t_{i+1}+1\right)$.

STEP 2. We set $d(t)=\left|p^{\prime}(t)-f(p(t), \alpha(z)(t), z(t))\right|$. For almost all $t \in\left(t_{i}, t_{i+1}\right)$ we have $d(t)=\left|f\left(p_{i}, \alpha(z)(t), z(t)\right)-f(p(t), \alpha(z)(t), z(t))\right| \leq L\left|p_{i}-p(t)\right| \leq L\left(\left|p_{i}-p\left(t_{i}\right)\right|+\right.$ $\left.\left|p(t)-p\left(t_{i}\right)\right|\right) \leq L \gamma\left(t_{i}+1\right)+L R\left(t-t_{i}\right)$. By Gronwall Lemma, we have

$$
\left|p(T)-x\left(T ; 0, x_{0}, \alpha(z), z\right)\right| \leq \exp (L T) \int_{0}^{T} d(t) d t .
$$

We have $\int_{t_{i}}^{t_{i+1}} d(t) d t \leq L \gamma\left(t_{i}+1\right)\left(t_{i+1}-t_{i}\right)+L R \int_{t_{i}}^{t_{i+1}}\left(t-t_{i}\right) d t=L \gamma\left(t_{i}+1\right)\left(t_{i+1}-\right.$ $\left.t_{i}\right)+L R 1 / 2\left(t_{i+1}-t_{i}\right)^{2}$ and in consequence

$$
\int_{0}^{T} d(t) d t \leq L \gamma(T+1) T+\frac{1}{2} L R T \cdot \operatorname{diam}(\Pi) .
$$

Taking $\gamma$ and diam(П) sufficiently small we obtain $z \in N_{0}$ such that

$$
\operatorname{dist}\left(x\left(T ; 0, x_{0}, \alpha(z), z\right), K\right)<\varepsilon \text {. }
$$

We deduce from Theorem 6 the time dependent case.

Corollary 7. Let $f:[0, T] \times \mathbf{R}^{n} \times Y \times Z \rightarrow \mathbf{R}^{n}, P:[0, T] \rightsquigarrow \mathbf{R}^{n}$ be as in Theorem 1 . We assume that for all $t \in(0, T), x \in P(t)$ we have

$$
\forall\left(n_{t}, n_{x}\right) \in N_{\operatorname{Graph}(P)}^{0}(t, x) \exists z \in Z \forall y \in Y\left\langle\left(n_{t}, n_{x}\right),(1, f(t, x, y, z))\right\rangle \leq 0 .
$$

Then for every $t_{0} \in(0, T), x_{0} \in P\left(t_{0}\right)$ we have

$$
\forall \varepsilon>0, \forall \alpha \in \Gamma_{t_{0}}, \exists z(\cdot) \in N_{t_{0}}, \operatorname{dist}\left(x\left(T ; t_{0}, x_{0}, \alpha(z), z\right), P(T)\right)<\varepsilon .
$$

Proof. Fix $t_{0} \in(0, T)$ and $x_{0} \in P\left(t_{0}\right)$. We set

$$
\widetilde{K}=\operatorname{Graph}(P) \cup[0, \infty) \times \mathbf{R}^{n}
$$

and

$$
\tilde{f}(t, x, y, z)= \begin{cases}0 & \text { for } t \leq 0 \\ \left(t / t_{0}\right)(1, f(t, x, y, z)) & \text { for } t \in\left(0, t_{0}\right), \\ (1, f(t, x, y, z)) & \text { for } t \in\left[t_{0}, T\right) \\ (1, f(T, x, y, z)) & \text { for } t \geq T\end{cases}
$$

$\widetilde{K}, \widetilde{f}$ satisfy the assumptions of Theorem 6 . We take $(t, x) \in \widetilde{K}$ and $(s, p) \in \mathbf{R} \times \mathbf{R}^{n}$ such that

$$
\|(t, x)-(s, p)\|=\operatorname{dist}((s, p), \widetilde{K}) .
$$

If $t=0$, then $\tilde{f}(t, x, y, z)=0$ and (11) holds true. If $t \in(0, T)$, then $(t, x)-(s, p) \in$ $N_{G \operatorname{raph}(P)}^{0}(t, x)$ and by $(14),(11)$ holds true.

If $t \geq T$, then

$$
\operatorname{dist}((s, p), \widetilde{K}) \leq \operatorname{dist}\left((s, p),[T, \infty) \times R^{n}\right) \leq\|(s, p)-(t, x)\| .
$$


Thus $p=x$ and $s \leq t$. Therefore

$$
\langle(s, p)-(t, x), \tilde{f}(t, x, y, z)\rangle=s-t \leq 0,
$$

for all $y, z$.

Now, we fix $\varepsilon>0$ and $\alpha \in \Gamma_{t_{0}}$. By Theorem 6 , there exists $z \in N_{t_{0}}$ such that

$$
\operatorname{dist}((t(T-\varepsilon), x(T-\varepsilon)), \widetilde{K})<\varepsilon,
$$

where $y(s)=(t(s), x(s))$ is the solution of the Cauchy problem

$$
\left\{\begin{array}{l}
y^{\prime}(s)=\tilde{f}(y(s), \alpha(z)(s), z(s)), \\
y\left(t_{0}\right)=\left(t_{0}, x_{0}\right) .
\end{array}\right.
$$

Obviously, $t(s)=s$ and $x(s)=x\left(s ; t_{0}, x_{0}, \alpha(z), z\right)$.

Since $\operatorname{dist}\left(\left(T-\varepsilon, x\left(T-\varepsilon ; t_{0}, x_{0}, \alpha(z), z\right)\right), \widetilde{K}\right)<\varepsilon$, there is $t_{1} \in(T-2 \cdot \varepsilon, T)$ such that $\left.\operatorname{dist}\left(x\left(T-\varepsilon ; t_{0}, x_{0}, \alpha(z), z\right)\right), P\left(t_{1}\right)\right)<\varepsilon$. Thus

$$
\begin{aligned}
\operatorname{dist}\left(x\left(T ; t_{0}, x_{0}, \alpha(z), z\right), P(T)\right) \leq & \left\|x\left(T ; t_{0}, x_{0}, \alpha(z), z\right)-x\left(T-\varepsilon ; t_{0}, x_{0}, \alpha(z), z\right)\right\| \\
& +\varepsilon+d_{H}\left(P\left(t_{1}\right), P(T)\right),
\end{aligned}
$$

which completes the proof.

REMARK 8. If $Z=\left\{z_{0}\right\}$ then the differential game reduces to the control system with dynamics given by $\widehat{f}(t, x, y)=f\left(t, x, y, z_{0}\right)$. Assume moreover that $\left\{f\left(t, x, y, z_{0}\right) \mid y \in Y\right\}$ is convex for every $t$ and $x$. Then, from (14)

$$
\forall y \in Y,\left(1, f\left(t, x, y, z_{0}\right)\right) \in \overline{\mathrm{co}}\left(T_{\operatorname{Graph}(P)}(t, x)\right)
$$

and (4) implies that

$$
\exists y \in Y,\left(1, f\left(t, x, y, z_{0}\right)\right) \in \overline{\mathrm{co}}\left(T_{\operatorname{Graph}(P)}(t, x)\right),
$$

which should motivate the title of the paper.

3. Applications to Hamilton-Jacobi-Isaacs equations. In [6] the notion of viscosity solution was introduced using smooth test functions. Equivalently, it can be done using some nonsmooth analysis tools. In [5] it has been done using super- and subdifferentials. We recall it adding the third equivalent formulation using normal cones to hipoand epigraphs of functions.

A function $u$ from an open subset $D \subset \mathbf{R}^{n}$ into $\mathbf{R}$ is said to be differentiable at $x_{0} \in D$, and $D u\left(x_{0}\right)=p_{0}$, if we have

$$
\lim _{x \rightarrow x_{0}} \frac{u(x)-u\left(x_{0}\right)-\left\langle p_{0}, x-x_{0}\right\rangle}{\left\|x-x_{0}\right\|}=0 .
$$

The subdifferential of $u$ at $x_{0}$ is the set, denoted by $\partial_{-} u\left(x_{0}\right)$, of $p_{0} \in \mathbf{R}^{n}$ such that

$$
\liminf _{x \rightarrow x_{0}} \frac{u(x)-u\left(x_{0}\right)-\left\langle p_{0}, x-x_{0}\right\rangle}{\left\|x-x_{0}\right\|} \geq 0 .
$$

The superdifferential of $u$ at $x_{0}$ is the set, denoted by $\partial_{+} u\left(x_{0}\right)$, of $p_{0} \in \mathbf{R}^{n}$ such that

$$
\limsup _{x \rightarrow x_{0}} \frac{u(x)-u\left(x_{0}\right)-\left\langle p_{0}, x-x_{0}\right\rangle}{\left\|x-x_{0}\right\|} \leq 0 .
$$


The epigraph of the function $u$ is the set, denoted by $\operatorname{Epi}(u)$, of $(x, v) \in \mathbf{R}^{n} \times \mathbf{R}$ such that $v \geq u(x)$. The hypograph of the function $u$ is the set, denoted by $\operatorname{Hyp}(u)$, of $(x, v) \in \mathbf{R}^{n} \times \mathbf{R}$ such that $v \leq u(x)$.

Proposition 1.1 in [5] and Proposition 5.2 in [11] are summarized in the following proposition.

Proposition 9. If $u: D \rightarrow \mathbf{R}$ is a continuous function and $x_{0} \in D, p \in \mathbf{R}^{n}$, then the following conditions are equivalent:

(i) $p \in \partial_{-} u\left(x_{0}\right)$ (resp. $\left.p \in \partial_{+} u\left(x_{0}\right)\right)$,

(ii) there exists $\varphi \in C^{1}(D)$ such that $u-\varphi$ has a local minimum (resp. maximum) at $x_{0}$ and $D \varphi\left(x_{0}\right)=p$,

(iii) $(p,-1) \in N_{\operatorname{Epi}(u)}^{0}\left(x_{0}, u\left(x_{0}\right)\right)\left(\operatorname{resp} .(-p, 1) \in N_{\operatorname{Hyp}(u)}^{0}\left(x_{0}, u\left(x_{0}\right)\right)\right)$.

Definition 10. A viscosity supersolution of Hamilton-Jacobi-Isaacs equation

$$
U_{t}+H\left(t, x, U_{x}\right)=0
$$

is a continuous function $U:[0, T] \times \mathbf{R}^{n} \rightarrow \mathbf{R}$ satisfying for every $\left(t_{0}, x_{0}\right) \in(0, T) \times \mathbf{R}^{n}$

$$
p_{t}+H\left(t, x, p_{x}\right) \leq 0, \quad \forall\left(p_{t}, p_{x},-1\right) \in N_{\operatorname{Epi}(U)}^{0}\left(t_{0}, x_{0}, U\left(t_{0}, x_{0}\right)\right) .
$$

A viscosity subsolution of Hamilton-Jacobi-Isaacs equation

$$
U_{t}+H\left(t, x, U_{x}\right)=0
$$

is a continuous function $U:[0, T] \times \mathbf{R}^{n} \rightarrow \mathbf{R}$ satisfying for every $\left(t_{0}, x_{0}\right) \in(0, T) \times \mathbf{R}^{n}$

$$
p_{t}+H\left(t, x, p_{x}\right) \geq 0, \quad \forall\left(-p_{t},-p_{x}, 1\right) \in N_{\mathrm{Hyp}(U)}^{0}\left(t_{0}, x_{0}, U\left(t_{0}, x_{0}\right)\right) .
$$

A function is a viscosity solution if it is both super- and subsolution.

The definition of viscosity solution can be equivalently formulated using sub- and super differentials or smooth test function (comp. Definition 1 and 2 in [5]). It is direct conlusion from Proposition 9.

Let $f$ be as in Theorem 1 and $g: \mathbf{R}^{n} \rightarrow \mathbf{R}$ be Lipschitz continuous. We define the value function $U:[0, T] \times \mathbf{R}^{n} \rightarrow \mathbf{R}$ by:

$$
U\left(t_{0}, x_{0}\right)=\sup _{\alpha \in \Gamma_{t_{0}}} \inf _{z \in N_{t_{0}}} g\left(x\left(T ; t_{0}, x_{0}, \alpha(z), z\right)\right)
$$

and Hamiltonian $H:[0, T] \times \mathbf{R}^{n} \times \mathbf{R}^{n} \rightarrow \mathbf{R}$ by

$$
H(t, x, p)=\min _{z \in Z} \max _{y \in Y}\langle f(t, x, y, z), p\rangle .
$$

Evans and Souganidis in [8] proved that $U$ is a viscosity solution of Isaacs equation (1) and is $U$ Lipschitz continuous. Our aim is to prove that if a Lipschitz continuous function $W:[0, T] \times \mathbf{R}^{n} \rightarrow \mathbf{R}$ is a viscosity solution of Isaacs equation and satisfies the condition $W(T, \cdot)=g(\cdot)$ then $W=U$.

We define the tube $H:[0, T] \rightsquigarrow \mathbf{R}^{n}$ by $H(t)=\{(x, u) \mid u \leq U(t, x)\}$. Obviously, we have $\operatorname{Graph}(H)=\operatorname{Hyp}(U)$. Since $U$ is Lipschitz continuous, then (16) is equivalent to

$$
\begin{aligned}
\forall\left(n_{t}, n_{x}, n_{u}\right) \in N_{\operatorname{Graph}(H)} & (t, x, U(t, x)) \forall z \in Z, \exists y \in Y \\
& \left\langle\left(n_{t}, n_{x}, n_{u}\right),(1, f(t, x, y, z), 0)\right\rangle \leq 0 .
\end{aligned}
$$

It means that $\tilde{f}(t, x, u, y, z)=(f(t, x, y, z), 0)$ satisfies (4) with respect to the tube $H$. 
If we define the tube $E:[0, T] \rightsquigarrow \mathbf{R}^{n}$ by $E(t)=\{(x, u) \mid u \geq U(t, x)\}$ then obviously $\operatorname{Graph}(E)=\operatorname{Epi}(U)$ and condition (15) can be equivalently formulated

$$
\begin{aligned}
\forall\left(n_{t}, n_{x}, n_{u}\right) \in N_{\operatorname{Graph}(E)} & (t, x, U(t, x)), \exists z \in Z, \forall y \in Y, \\
& \left\langle\left(n_{t}, n_{x}, n_{u}\right),(1, f(t, x, y, z), 0)\right\rangle \leq 0 .
\end{aligned}
$$

It means that $\tilde{f}(t, x, u, y, z)=(f(t, x, y, z), 0)$ satisfies $(14)$ with respect to the tube $E$.

We have the following:

Proposition 11. If a Lipschitz continuous function $W:[0, T] \times \mathbf{R}^{n} \rightarrow \mathbf{R}$ is a viscosity subsolution of (1) and $W(T, \cdot)=g(\cdot)$ holds true, then we have $W\left(t_{0}, x_{0}\right) \leq U\left(t_{0}, x_{0}\right)$, for every $\left(t_{0}, x_{0}\right) \in[0, T] \times R^{n}$.

Proof. We consider the Cauchy problem

$$
\left\{\begin{array}{l}
\left(x^{\prime}(t), v^{\prime}(t)\right)=(f(t, x(t), y(t), z(t)), 0) \quad \text { for a.e. } t \in[0, T] \\
\left(x\left(t_{0}\right), v\left(t_{0}\right)=\left(x_{0}, W\left(t_{0}, x_{0}\right)\right) .\right.
\end{array}\right.
$$

If $(x(t), v(t))$ is a solution of the above Cauchy problem then $x(t)=x\left(t ; t_{0}, x_{0}, y(\cdot), z(\cdot)\right)$ and $v(t) \equiv W\left(t_{0}, x_{0}\right)$. By Viability Theorem and assumption (16) we obtain

$$
\exists \alpha \in \Gamma_{t_{0}} \forall z(\cdot) \in N_{t_{0}} \forall t \in\left[t_{0}, T\right]\left(x\left(t ; t_{0}, x_{0}, \alpha(z), z\right), W\left(t_{0}, x_{0}\right)\right) \in H(t) .
$$

This gives

$$
\exists \alpha \in \Gamma_{t_{0}} \forall z(\cdot) \in N_{t_{0}} W\left(t_{0}, x_{0}\right) \leq g\left(x\left(T ; t_{0}, x_{0}, \alpha(z), z\right)\right)
$$

and consequently $W\left(t_{0}, x_{0}\right) \leq U\left(t_{0}, x_{)}\right)$, which completes the proof.

Proposition 12. If a Lipschitz continuous function $W:[0, T] \times \mathbf{R}^{n} \rightarrow \mathbf{R}$ is a viscosity supersolution of $(1)$ and $W(T, \cdot)=g(\cdot)$ holds true, then we have

$$
U\left(t_{0}, x_{0}\right) \leq W\left(t_{0}, x_{0}\right), \quad \text { for every }\left(t_{0}, x_{0}\right) \in[0, T] \times R^{n} .
$$

Proof. By Corollary 7 and (16), we have

$$
\forall \varepsilon>0, \forall \alpha \in \Gamma_{t_{0}}, \exists z \in N_{t_{0}}, \operatorname{dist}\left(\left(x\left(T ; t_{0}, x_{0}, \alpha(z), z\right), W\left(t_{0}, x_{0}\right)\right)<\varepsilon .\right.
$$

Thus

$$
\forall \varepsilon>0, \forall \alpha \in \Gamma_{t_{0}}, \exists z \in N_{t_{0}}, g\left(x\left(T ; t_{0}, x_{0}, \alpha(z), z\right)\right) \leq W\left(t_{0}, x_{0}\right)+(L+1) \varepsilon,
$$

where $L$ is a Lipschitz constant for $g$. Therefore

$$
\forall \varepsilon>0 \sup _{\alpha \in \Gamma_{t_{0}}} \inf _{z \in N_{t_{0}}} g\left(x\left(T ; t_{0}, x_{0}, \alpha(z), z\right)\right) \leq W\left(t_{0}, x_{0}\right)+(L+1) \varepsilon,
$$

which completes the proof.

As a corollary we obtain the following

THEOREM 13. If a Lipschitz continuous function $W:[0, T] \times \mathbf{R}^{n} \rightarrow \mathbf{R}$ is a viscosity solution of the equation $W_{t}+H\left(t, x, W_{x}\right)=0$ and $W(T, \cdot)=g(\cdot)$ then $W=U$.

Concluding remarks. If the right-hande side $f(t, x, y, z)$ is "only" measurable with respect to the time, then the Hamiltonian $H(t, x, p)$ determined by $f$ is also "only" measurable with respect to " $t$ ". In spite of this the value function $U$ can be correctly define. If the right-hand sides $f_{1}, f_{2}$ satisfies the condition

$$
f_{1}(t, x, y, z)=f_{2}(t, x, y, z) \quad \text { for every } t \in C,(x, y, z) \in \mathbf{R} \times Y \times Z
$$


where $C$ is a full measure subset of $[0, T]$, then $U_{1}=U_{2}$, where $U_{1}, U_{2}$ are value function determined by $f_{1}, f_{2}$ respectively. But Hamiltonians $H_{1}, H_{2}$ can be different on the set $([0, T] \backslash C) \times \mathbf{R}^{n} \times Y \times Z$. So, in the definition of viscosity solutions seems to be reasonable to require that conditions (16) and (15) hold true only for every $(t, x) \in C \times \mathbf{R}^{n}$. Such a modification in the definition of viscosity solution was provided by Frankowska, Plaskacz, Rzeżuchowski in [11] for Hamiltonians that are convex with respect to " $p$ ". To adopt the method presented in the paper to the measurable case one have to generalize Theorem 1 and Corollary 7. It will be done in the forthcoming paper of the authors.

\section{References}

[1] J.-P. Aubin, Viability Theory, Birkhäuser, Boston, Basel, Berlin (1991).

[2] J.-P. A ubin and A. Cellina, Differential Inclusions, Springer-Verlag (1984).

[3] J.-P. Aubin and H. Frankowska, Set-Valued Analysis, Birkhäuser, Boston, Basel, Berlin (1990).

[4] P. Cardaliaguet, Domaines discriminant en jeux différentiels, Ph.D. Thesis, Université Paris Dauphine (1992).

[5] M. G. Crandall, L. C. Evans and P. L. Lions, Some properties of viscosity solutions of Hamilton-Jacobi equations, Trans. Amer. Math. Soc. 282, 487-502.

[6] M. G. Crandall and P. L. Lions, Viscosity solutions of Hamilton-Jacobi equations, Trans. Amer. Math. Soc. 277 (1983), 1-42.

[7] R. J. Elliott and N. J. Kalton, The existence of value in differential games, Mem. Amer. Math. Soc. 126 (1972).

[8] L. C. Evans and P. E. Souganidis, Differential games and representation formulas for solutions of Hamilton-Jacobi-Isaacs equations, Indiana Univ. Math. J. 33 (1984), 773-797.

[9] H. Frankowska, Lower semicontinuous solutions of Hamilton-Jacobi-Bellman equations, SIAM J. Control And Optimization 31 (1993), 257-272.

[10] H. Frankowska and S. Plaskacz, A measurable - upper semicontinuous viability theorem for tubes, Nonlinear Analysis TMA. (to appear).

[11] H. Frankowska, S. Plaskacz and T. Rzeżuchowski, Théorèmes de viabilité mesurables et l'équation d'Hamilton-Jacobi-Bellman, Comptes-Rendus de l'Académie des Sciences, Paris, Série 1 (1992).

[12] H. Frankowska, S. Plaskacz and T. Rzeżuchowski, Measurable viability theorems and Hamilton-Jacobi-Bellman equation, J. Diff. Eqs. 116 (1995), 265-305.

[13] R. T. Rockafellar, Proximal subgradients, marginal values, and augmented Lagrangians in nonconvex optimization, Math. of Oper. Res. 6 (1981), 424-436.

[14] E. Roxin, The axiomatic approach in differential games, J. Optim. Theory Appl. 3 (1969), 153-163.

[15] P. P. Varaiya, The existence of solutions to a diffrential game, SIAM J. Control Optim. 5 (1967), 153-162. 